\title{
Reconfiguration of a Primary Three-Phase Three-Wire Distribution Network with Unbalanced Loads
}

\author{
Irina Golub ${ }^{1, *}$, Oleg Voitov ${ }^{1}$, Evgeni Boloev ${ }^{1}$ and Lyudmila Semenova ${ }^{1}$ \\ ${ }^{1}$ Energy Systems Institute of Siberian Branch of the Russian Academy of Sciences, Department of Electric Power System , 664033 \\ Lermontov str., 130, Irkutsk, Russia
}

\begin{abstract}
The paper deals with the problem of reconfiguration of a primary three-phase three-wire distribution network with unbalanced loads following the criterion of power loss minimization. The authors propose modification of a well-proven algorithm the need for which has been necessitated by using an unbalanced network of power losses and currents in it for reconfiguration. For computing the parameters mentioned, a program for load flow computation in the balanced network is used for modeling an unbalanced network as a single-line equivalent scheme. Efficiency of proposed approaches has been confirmed by results of reconfiguration of a test distribution network.
\end{abstract}

\section{Introduction}

Reconfiguration of a primary distribution network is, first of all, intended to ensure reliable power supply for consumers, and is an efficient method of reducing power losses that in the distribution networks may be as high as $10-13 \%$. A primary distribution network powers the secondary low-voltage distribution network that is directly related to loads that may be single-phase, twophase and three-phase ones.

Loads unbalance in the secondary distribution network causes three-phase unbalances of loads in the primary network, unbalances of bus voltages and losses in transmission lines, and may also cause current overloads in the lines. The present paper considers the problem of reducing the impact of loads unbalance on losses in the distribution network through the reconfiguration procedure.

It should be noted that unbalance of configuration of a three-phase four-wire network often discussed in foreign literature is not typical of Russian primary distribution networks and, therefore, is not considered here, and the network is modeled as a three-phase threewire one. Analysis of an unbalanced three-phase primary network requires both its three-line representation, and development of a reconfiguration algorithm that would take into account peculiarities of such a network.

Development of optimization algorithms for primary network reconfiguration is discussed in many papers that use a single-line representation of a three-phase network implying its balance, and setting the load equal to total load of phases.

Optimization heuristic algorithms of configuration, such as genetic [1], tabu search [2], ant colony $[3,4]$, harmony search [5], and particle swarm optimization [6] algorithms have been widely used, but their computational burden impedes their real-time use.

Let us give brief characteristic of a configuration problem. A primary distribution network operates as an open network owing to availability of normally opened tie-switches and normally closed sectionalizing switches. Closing of a tie-switch and opening of the corresponding sectionalizing switch results in a new radial network configuration; a procedure for configuration change that is used for ensuring the power supply reliability and reduction of losses in the network is referred to as 'reconfiguration'.

For reconfiguring the primary network the authors developed an algorithm [7] based on the known in the theory of graphs methods for determination (on the graph of a network) of maximum spanning tree and a mix of branches of independent loops by their chords the line switches are associated with. That algorithm is included into the fast operating software for load flow calculation, SDO-7 [8].

This algorithm was used for reconfiguring a large number of test and actual distribution networks, which allowed identification of a large number of factors affecting the results of reconfiguration. Properties identified for a single-line model of the network shall work within a three- line model of the network.

In particular, it was shown that integration of renewable energy into a distribution network has a higher impact on losses reduction than reconfiguration, whereas reconfiguration of a network with renewable energy sources requires a larger amount of switching than reconfiguration in the network without renewable energy sources [9].

The authors have demonstrated the possibility of selecting such a permanent configuration of a 
distribution network where power losses not only within a day but within a week with maximum load and a week with minimum load will be close to losses at optimum hourly reconfiguration [10].

Ref. [10] also shows that information on the mix of loop branches obtained during reconfiguration algorithm operation can be used for choosing the tie-switches that ensure both conditions restoration after emergency disconnection of a sectionalizing switch and accelerate this process, especially if a tie-switch is remotely controlled.

The paper is arranged in the following way.

Section 2 analyses the possibility of using the load flow calculation program for calculating the conditions of a three-phase three-wire network with unbalanced loads, which required conversion of a three-wire model of the network into a single-line one.

Section 3 gives expressions for determination of power losses and currents in the phases of a three-wire line that are required for operation of a reconfiguration algorithm.

Section 4 gives a reconfiguration algorithm [7] and changes incorporated for the purpose of its use in a network with unbalanced loads.

Section 5 demonstrates the efficiency of using a modified reconfiguration algorithm for a network with unbalanced loads on the example of a test network [10].

\section{Three-phase modeling of a primary distribution network}

Analysis of operating conditions of an unbalanced primary distribution network required its three-phase three-wire modeling.

Alternatively, load flow of a three-phase network with unbalanced loads can be calculated using programs developed for a single-line model of a balanced network that requires reduction of an unbalanced three-phase three-wire network to its single-line representation.

According to this approach, a section of a three-phase three-wire feeder in a distribution network is modeled as a single-line equivalent network $[11,12]$ whose parameters are defined by elements of a nodal admittance matrix $Y_{a b c}=Z_{a b c}^{-1}$, where $Z_{a b c}$ is a matrix of mutual and self impedances of section's branches in phase coordinates [13] without account of ground shunt capacitance of phase wires.

Under the lack of information on mutual and self impedances the matrix $Z_{a b c}$ is computed as

$$
Z_{a b c}=S D S^{-1}
$$

where $S$ is a matrix of transition of symmetry components to phase coordinates; $D$ is a diagonal matrix of reference values of impedances of positive, negative and zero sequences.

Let us consider the principle of reducing a threephase three-wire feeder between buses $i-j$ whose mutual and self impedances are shown in Fig. 1, to its single-line representation.

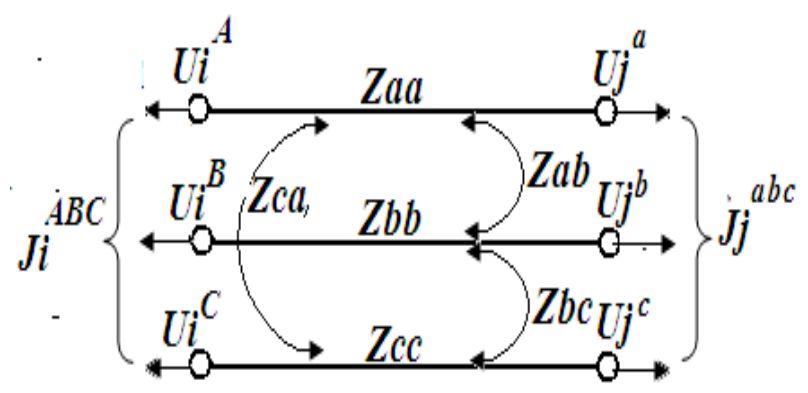

Fig. 1. Impedances of a three-phase feeder section.

Equations for currents balance in the initial $i$ and final $j$ nodes of the section $i-j$ can be put down as

$$
\begin{aligned}
& J_{i}^{A B C}+Y_{a b c}\left(U_{i}^{A B C}-U_{j}^{a b c}\right)=0 \\
& J_{j}^{a b c}-Y_{a b c}\left(U_{i}^{A B C}-U_{j}^{a b c}\right)=0
\end{aligned}
$$

where $J_{i}^{A B C}, J_{j}^{a b c}, U_{i}^{A B C}, U_{j}^{a b c}$ are column matrices of phase nodal currents and voltages in the nodes of section $i-j$. A matrix of nodal impedances (2) can be represented as

$$
Y_{a b c}=\left(\begin{array}{lll}
y_{11} & y_{12} & y_{13} \\
y_{21} & y_{22} & y_{23} \\
y_{31} & y_{32} & y_{33}
\end{array}\right) .
$$

Let us put down equations (2) as matrix form for the node-voltage equation

$$
\left(\begin{array}{c}
J_{i}^{A B C} \\
J_{j}^{a b c}
\end{array}\right)=\left(\begin{array}{cc}
-Y_{a b c} & Y_{a b c} \\
Y_{a b c} & -Y_{a b c}
\end{array}\right) \cdot\left(\begin{array}{l}
U_{i}^{A B C} \\
U_{j}^{a b c}
\end{array}\right)=Y\left(\begin{array}{l}
U_{i}^{A B C} \\
U_{j}^{a b c}
\end{array}\right) .
$$

Marking of columns and rows of the matrix $Y$ and values of its elements have the form

$$
Y=\left(\begin{array}{ccccccc} 
& A & B & C & a & b & c \\
A & -y_{11} & -y_{12} & -y_{13} & y_{11} & y_{12} & y_{13} \\
B & -y_{21} & -y_{22} & -y_{23} & y_{21} & y_{22} & y_{23} \\
C & -y_{13} & -y_{23} & -y_{33} & y_{31} & y_{32} & y_{33} \\
a & y_{11} & y_{12} & y_{13} & -y_{11} & -y_{11} & -y_{11} \\
b & y_{21} & y_{22} & y_{23} & -y_{21} & -y_{22} & -y_{23} \\
c & y_{31} & y_{32} & y_{33} & -y_{31} & -y_{32} & -y_{33}
\end{array}\right) .
$$

Matrix $Y$ is a nodal admittance matrix; it is a symmetry matrix and a sum of elements of its rows and columns equals zero. When constructing a network graph on the base of a matrix of nodal admittances, only its non-diagonal elements are used. Such a graph (Fig. 2 ) is a desired single-line model the section $i-j$ by a 
three-phase three-wire line. Admittance of each graph's branch equals a value of the corresponding element of matrix $Y$ taken with the negative sign. For example, admittance of tie A-a equals $-y_{11}$.

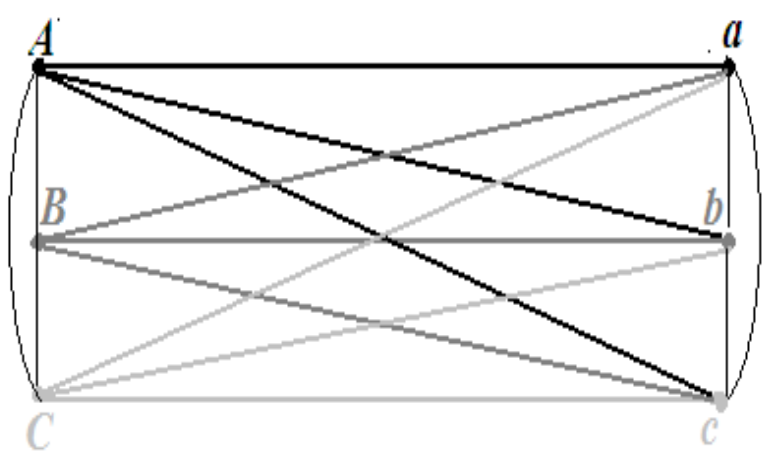

Fig. 2. A graph of a single-line model the section of a threephase three-wire line.

\section{Calculation of load flow in the unbalanced distribution network}

Load flow in the unbalanced three-phase three-wire distribution network in the course of its reconfiguration on the base of a single-line model was calculated using SDO-7 software [8] designed for computing the balanced network.

Such computations required modification of the software that allowed:

- Construction of a single-line equivalenting scheme for every section of a three-phase three-wire line and interconnection of the sections to obtain a single-line scheme for the network equivalenting.

- Input of data on phase loads and phase voltages of a power source into the pattern obtained;

- Solution of the system of nodal power balance equations by Newton's method to determine the complex values of nodal voltages [8];

- Calculation of phase values of active and reactive power flows, current and power losses for each section $i-j$ of a three-phase line.

For example, equations for active and reactive power flows of the phase $a$ at the beginning of section $i-j$ those are determined as a sum of load flows in the adjacent node $A$ ties $A B, A C, A a, A b, A c$, similarly to [12], can be represented as

$$
\begin{gathered}
P_{i j}^{a}=P_{i j}^{A B}+P_{i j}^{A C}+P_{i j}^{A a}+P_{i j}^{A b}+P_{i j}^{A c}= \\
=\left(U_{i}^{A}\right)^{2} g_{i j}^{A B}-U_{i}^{A} U_{i}^{B}\left(g_{i j}^{A B} \cos \left(\delta_{i}^{A}-\delta_{i}^{B}\right)+b_{i j}^{A B} \sin \left(\delta_{i}^{A}-\delta_{i}^{B}\right)\right) \\
+\left(U_{i}^{A}\right)^{2} g_{i j}^{A C}-U_{i}^{A} U_{i}^{C}\left(g_{i j}^{A C} \cos \left(\delta_{i}^{A}-\delta_{i}^{C}\right)+b_{i j}^{A C} \sin \left(\delta_{i}^{A}-\delta_{i}^{C}\right)\right) \\
+\left(U_{i}^{A}\right)^{2} g_{i j}^{A a}-U_{i}^{A} U_{j}^{a}\left(g_{i j}^{A a} \cos \left(\delta_{i}^{A}-\delta_{j}^{a}\right)+b_{i j}^{A a} \sin \left(\delta_{i}^{A}-\delta_{j}^{a}\right)\right) \\
+\left(U_{i}^{A}\right)^{2} g_{i j}^{A b}-U_{i}^{A} U_{j}^{b}\left(g_{i j}^{A b} \cos \left(\delta_{i}^{A}-\delta_{j}^{b}\right)+b_{i j}^{A b} \sin \left(\delta_{i}^{A}-\delta_{j}^{b}\right)\right) \\
+\left(U_{i}^{A}\right)^{2} g_{i j}^{A c}-U_{i}^{A} U_{j}^{c}\left(g_{i j}^{A c} \cos \left(\delta_{i}^{A}-\delta_{j}^{c}\right)+b_{i j}^{A c} \sin \left(\delta_{i}^{A}-\delta_{j}^{c}\right)\right) .
\end{gathered}
$$

$$
\begin{gathered}
Q_{i j}^{a}=Q_{i j}^{A B}+Q_{i j}^{A C}+Q_{i j}^{A a}+Q_{i j}^{A b}+Q_{i j}^{A c}= \\
=-\left(U_{i}^{A}\right)^{2} b_{i j}^{A B}-U_{i}^{A} U_{i}^{B}\left(g_{i j}^{A B} \sin \left(\delta_{i}^{A}-\delta_{i}^{B}\right)-b_{i j}^{A B} \cos \left(\delta_{i}^{A}-\delta_{i}^{B}\right)\right) \\
-\left(U_{i}^{A}\right)^{2} b_{i j}^{A C}-U_{i}^{A} U_{i}^{C}\left(g_{i j}^{A C} \sin \left(\delta_{i}^{A}-\delta_{i}^{C}\right)-b_{i j}^{A C} \cos \left(\delta_{i}^{A}-\delta_{i}^{C}\right)\right) \\
-\left(U_{i}^{A}\right)^{2} b_{i j}^{A C}-U_{i}^{A} U_{i}^{C}\left(g_{i j}^{A C} \sin \left(\delta_{i}^{A}-\delta_{i}^{C}\right)-b_{i j}^{A C} \cos \left(\delta_{i}^{A}-\delta_{i}^{C}\right)\right) \\
-\left(U_{i}^{A}\right)^{2} b_{i j}^{A a}-U_{i}^{A} U_{j}^{a}\left(g_{i j}^{A a} \sin \left(\delta_{i}^{A}-\delta_{j}^{a}\right)-b_{i j}^{A a} \cos \left(\delta_{i}^{A}-\delta_{j}^{a}\right)\right) \\
-\left(U_{i}^{A}\right)^{2} b_{i j}^{A b}-U_{i}^{A} U_{j}^{b}\left(g_{i j}^{A b} \sin \left(\delta_{i}^{A}-\delta_{j}^{b}\right)-b_{i j}^{A b} \cos \left(\delta_{i}^{A}-\delta_{j}^{b}\right)\right) \\
-\left(U_{i}^{A}\right)^{2} b_{i j}^{A c}-U_{i}^{A} U_{j}^{c}\left(g_{i j}^{A c} \sin \left(\delta_{i}^{A}-\delta_{j}^{c}\right)-b_{i j}^{A c} \cos \left(\delta_{i}^{A}-\delta_{j}^{c}\right)\right)
\end{gathered}
$$

An equation for computing the power losses in the phases for active power flows (similarly to a reactive power flow) of the phase $a$ at the end of section $i-j$, that is determined as a sum of load flows from the opposite ends of the adjacent to the node $a$ ties $a b, a c, a A, a B, a C$ can be put down as

$$
\begin{gathered}
P_{j i}^{a}=P_{j i}^{a b}+P_{j i}^{a c}+P_{j i}^{a A}+P_{j i}^{a B}+P_{j i}^{a C}= \\
=-\left(U_{j}^{a}\right)^{2} g_{i j}^{a b}+U_{j}^{a} U_{j}^{b}\left(g_{i j}^{a b} \cos \left(\delta_{j}^{a}-\delta_{j}^{b}\right)-b_{i j}^{a b} \sin \left(\delta_{j}^{a}-\delta_{j}^{b}\right)\right) \\
-\left(U_{j}^{a}\right)^{2} g_{i j}^{a c}+U_{j}^{a} U_{j}^{c}\left(g_{i j}^{a c} \cos \left(\delta_{j}^{a}-\delta_{j}^{c}\right)-b_{i j}^{a c} \sin \left(\delta_{j}^{a}-\delta_{j}^{c}\right)\right) \\
-\left(U_{j}^{a}\right)^{2} g_{i j}^{a A}+U_{j}^{a} U_{i}^{A}\left(g_{i j}^{a A} \cos \left(\delta_{j}^{a}-\delta_{i}^{A}\right)-b_{i j}^{a A} \sin \left(\delta_{j}^{a}-\delta_{i}^{A}\right)\right) \\
-\left(U_{j}^{a}\right)^{2} g_{i j}^{a B}+U_{j}^{a} U_{i}^{B}\left(g_{i j}^{a B} \cos \left(\delta_{j}^{a}-\delta_{i}^{B}\right)-b_{i j}^{a B} \sin \left(\delta_{j}^{a}-\delta_{i}^{B}\right)\right) \\
-\left(U_{j}^{a}\right)^{2} g_{i j}^{a C}+U_{j}^{a} U_{i}^{C}\left(g_{i j}^{a C} \cos \left(\delta_{j}^{a}-\delta_{i}^{C}\right)-b_{i j}^{a C} \sin \left(\delta_{j}^{a}-\delta_{i}^{C}\right)\right) .
\end{gathered}
$$

Active power losses and a current module, for example, for the phase $a$, of the section $i-j$ of a three-wire line are determined as

$$
\begin{gathered}
\Delta P_{i j}^{a}=P_{i j}^{a}-P_{j i}^{a}, \\
I_{i j}^{a}=\sqrt{\left(P_{i j}^{a}\right)^{2}+\left(Q_{i j}^{a}\right)^{2}} / U_{i}^{A} .
\end{gathered}
$$

\section{A reconfiguration algorithm for primary distribution network}

Peculiarity of the applied reconfiguration algorithm is neglect of information on the specified mix of opened tie-switches in the distribution network that at the commencement of the algorithm operation are assumed to be closed. Since losses in the closed network are, as a rule, lower that in the opened network, losses in the network will be minimal.

In the course of reconfiguration it is advisable that losses in the open network should remain close to losses in the closed network.

This condition will be observed if a maximum spanning tree with minimum currents in chords is constructed on the network graph using an algorithm [14] that is well-known in the theory of graphs. As a result, the graph's branches will belong either to branches of a tree or to chords of independent loops is associated with. Branches of independent loops can be 
determined by their chords using a graph theory algorithm proposed in [15].

A single-line network model similar to that in the initial algorithm [6] was used in the modified reconfiguration algorithm for unbalanced three-phase three-wire network when constructing a spanning tree, and when determining chords and branches belonging to every independent loop. Difference between a modified algorithm and an initial algorithm is that the expressions (6)-(10) were used for computation of load flows, losses and total values of currents magnitude of phase $a, b, c$ serving as weights when constructing a maximum spanning tree.

The first step of a reconfiguration algorithm consisting of two steps tends to obtain an opened graph (for which active power losses will be minimum) by opening the chords with minimum currents. For that purpose the following procedures are to be performed:

1 After closing all the tie-switches the load flows are to be computed, total losses and active power losses in phases $a, b, c$. are to be determined.

2 Then chords in independent loops are to be determined.

3 Loops are sorted as per ascending currents in the related chords.

4. A chord with minimum current opens and load flow is calculated.

5. Then we go over to Item 2 and after opening all the chords we go over to the second step of the algorithm.

As a result, after the first stage of reconfiguration (with the minimum computational effort) a new mix of opened chords is determined that correspond to tieswitches; power losses in the network at this mix are, as a rule, much lower than power losses at an arbitrary mix of normally opened tie-switches.

The second step of the algorithm consists in studying the potential for reducing losses in the network by replacing the chords opened at the first step by branches of a tree in the loop corresponding to the chord.

1. The analysis starts with closing the chord of the first loop out of those considered at the previous step.

2. Then the mix of loop's branches is determined to be followed by load flows and losses calculation.

3. Nodes whose power exceeds two are singled out in the closed loop on the left and right of the chord nodes.

4. Serial opening of branches located between singled out nodes and nodes of a chord is simulated. If there are no nodes whose power exceeds two in the loop, then opening of all the loop's branches is modeled.

5. Calculation of load flows and total losses is to follow. 6. A previous or a new chord with minimum total losses opens.

The reconfiguration algorithm is noted for high speed of making the decision with high accuracy of load flow calculation.

\section{Experimental studies}

Let us illustrate the use of software for load flow calculation in the unbalanced three-phase three-wire network, for a modified reconfiguration algorithm for selecting the configuration of a primary distribution network following the losses minimization criterion being integrated into it. A 33-node network known as a network proposed by M.E. Baran [5 ], Fig. 3, was taken as an example.

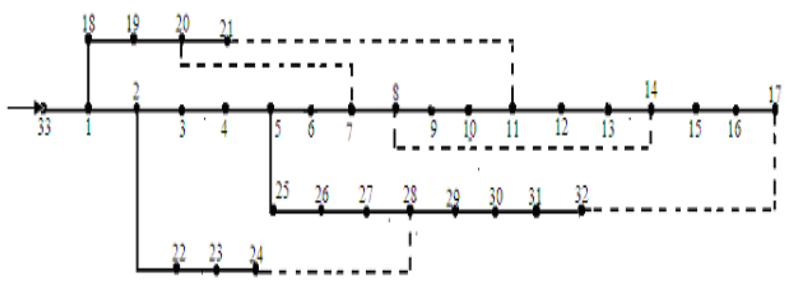

Fig. 3. Diagram of a 33-node distribution network; dotted lines denote branches with tie-switches.

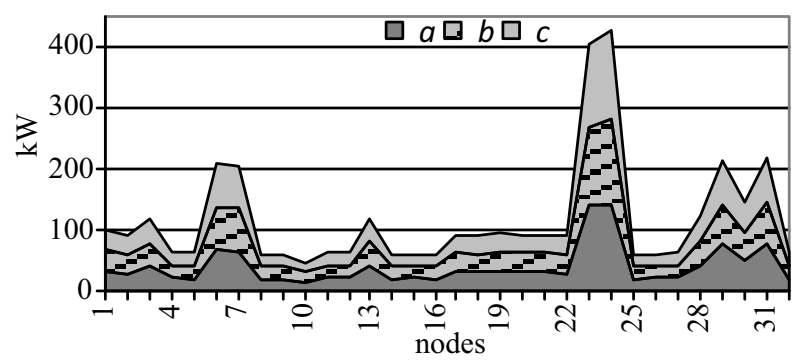

Fig. 4. Active loads.

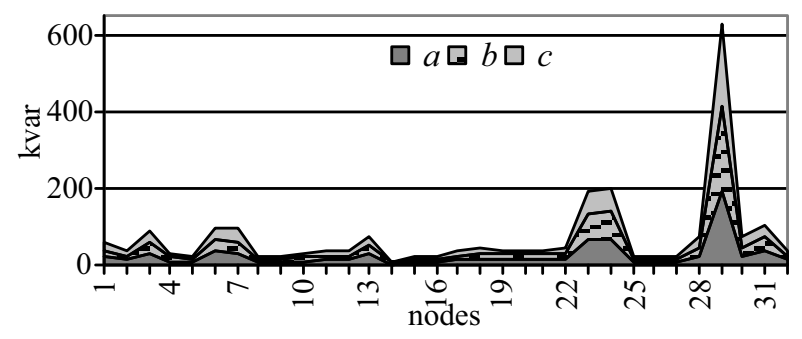

Fig. 5. Reactive loads.

Active and reactive unbalanced phase loads shown in Figs. 4 and 5 were obtained by adding $10 \%$ of phase deviations to their average values that were generated by a random number generator. Total power of active loads of phases $a, b, c$ equals $1250.4 \mathrm{~kW}, 1230.3 \mathrm{~kW}$ and $1269.6 \mathrm{~kW}$, accordingly, whereas total reactive loads of phases $a, b, c$ make 750 kvar, 790.7 kvar and 775.7 kvar .

Active power losses in phases $a, b, c$ of distribution network at opened tie-switches shown in Fig. 3 were found to be equal to $66.87 \mathrm{~kW}, 74.16 \mathrm{~kW}, 77.16 \mathrm{~kW}$, and total losses were as high as $218.19 \mathrm{~kW}$. After closing the tie-switches the losses in phases reduced down to $46.68 \mathrm{~kW}, 51.76 \mathrm{~kW}, 53.47 \mathrm{~kW}$, and total losses fell down to $151.91 \mathrm{~kW}$.

The network reconfiguration process is shown in Figs, 6 and 7 that evidence change in the active power losses at each phase and total losses at the first and second steps of the reconfiguration algorithm.

Power losses in the network with closed switches at the first stage of reconfiguration are input ones. Branches with tie-switches whose opening leads to 
minimum increase of total power losses are determined at each (out of five) iteration. Such switches opened in consequence at the first stage of reconfiguration where total losses increased from $151.91 \mathrm{~kW}$ to $170.31 \mathrm{~kW}$ were switches in sections 10-11, 13-14, 17-32, 7-20, 2728.

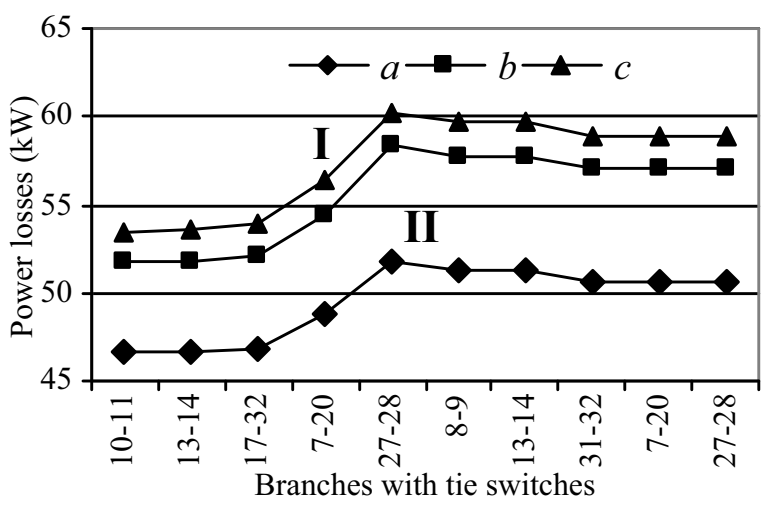

Fig. 6. Changes in the power losses in phases $a, b, c$ at the first I and second II stages of reconfiguration.

At the second stage of reconfiguration we analyzed the possibility of reducing the losses by closing the tieswitches determined at the first step and by opening the sectionalizing switches. There are two options to reduce losses: by closing the switches 10-11 and opening the switch 8-9 total losses dropped down to $168.65 \mathrm{~kW}$, and down to $166.46 \mathrm{~kW}$ by closing the switches 17-32 and opening the switches 31-32.

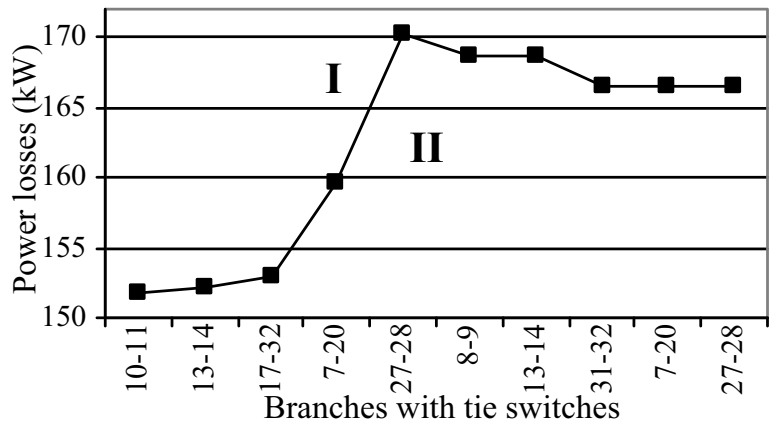

Fig. 7. Changes in the total power losses in phases at the first I and second II stages of the network reconfiguration, Fig. 3.

Reduction of voltage losses along with reduction of power losses is another important result of reconfiguration of a distribution network with asymmetry loads. This is confirmed by the results given in Figs. 8 and 9 that show voltage modules in the network before and after reconfiguration that allowed one to increase minimum values of voltages in the network nodes by $2 \mathrm{~kW}$ and higher, the voltage in the node being equal to $7.3 \mathrm{~kW}$.

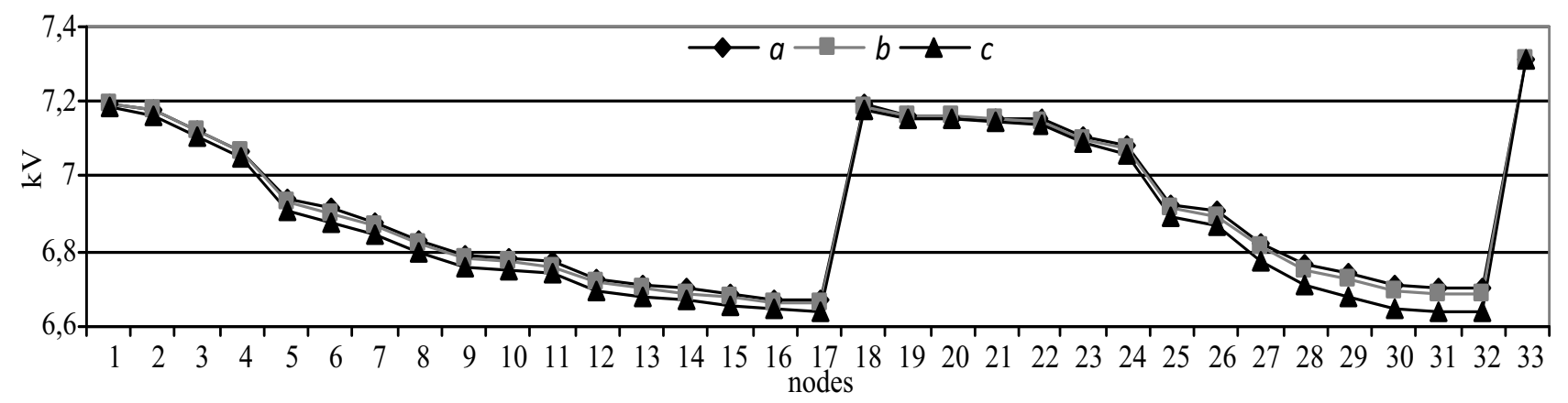

Fig. 8. Voltages in the nodes of phases $a, b, c$ before reconfiguration.

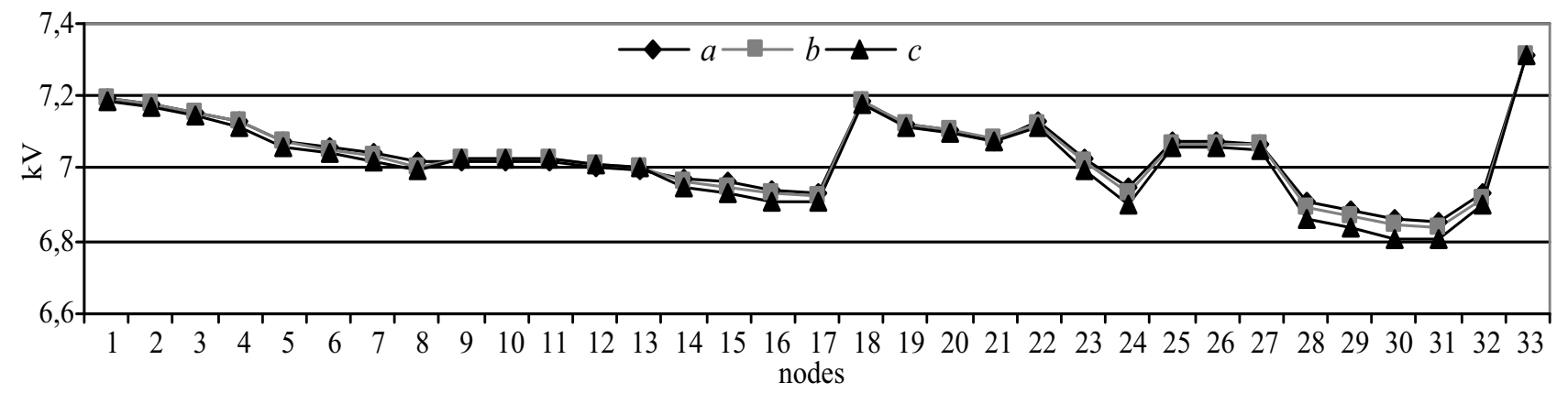

Fig. 9. Voltages in the nodes of phases $a, b, c$ after reconfiguration.

This study was supported by the project III.17.4.2 of the fundamental research program SB RAS, reg. No. AAAA-A17117030310438-1. 


\section{References}

1. M.A.N. Guimarães, C.A. Castro, R. Romero, IET Generation, Transmission \& Distribution 4 (11), 1213 (2010)

2. B.R.P. Junior; A.M. Cossi; J. Contreras, J.R.S. Mantovani, IET Generation, Transmission \& Distribution 8 (1), 35 (2014)

3. A.Y. Abdelaziz, R.A. Osama, S.M. El-Khodary, IET Generation, Transmission \& Distribution 6 (2), 176 (2012)

4. A. Kavousi-Fard, T. Niknam, A. Khosravi International Journal of Electrical Power \& Energy Systems 55, 680 (2014)

5. W.C. Wu, M.S. Tsai, IEEE Trans. on Power Systems 26(3), 1591 (2011)

6. I.I. Golub, O.N. Voitov, E.V. Boloev, Acta Energetica 31(2), 57 (2017)

7. V O.N. Voitov, System studies in energy. Retrospective of scientific schools at SEI-ISEM, 324 (Nauka, Novosibirsk, 2010)
8. I.I. Golub, O.N. Voitov, E.V. Boloev, L.V. Semenova Energy Systems Research 1 (1), 74 (2018)

9. I.I. Golub, O.N. Voitov, E.V. Boloev, L.V. Semenova, E3S Web of Conferences, 58, 1 (2018).

10. M.E Baran, F.F. Wu, IEEE Trans. Power Del. 4, 1401 (1989)

11. T.-H. Chen, M.-S. Chen, K.-J. Hwang, P. Kotas, E. A. Chebli IEEE Trans. Power Del. 6 (3), 1146 (1991)

12. C. N. Lu, J. H. Teng, W. H. E. Liu, IEEE Trans. on Power Systems 10 (1), 229 (1995)

13. D P Kothari, I J Nagrath, Modern Power System Analysis (Tata McGraw Hill Education private Limited, New Delhi 2009)

14. . E. Mainika, Algorithms for optimization on networks and graphs (M. Dekker, New York, 1978)

15. S.K. Gursky, Algorithmization of condition control problems for bulk power systems (Nauka i tekhnika, Minsk, 1977) 